\title{
Puberty and social brain development
}

\section{Abstract}

Puberty is characterized by substantial change in many areas of development, including hormonal, physical, neuronal, psychological and social. In this chapter, we outline how puberty, and its underlying hormonal and physical changes, might elicit a sensitive period for the development of the social brain. The literature to date suggests that pubertal development is relevant for the structural development of the social brain, partly in interaction with sex. Functionally, puberty might render the brain more sensitive to social information. However, methodological issues relating to sample size, study design and analysis, limit the possibility of drawing more specific conclusions. Apart from overcoming these methodological problems, future research should focus on individual differences in pubertal processes and their relevance to social brain development, as well as examining the mechanisms from pubertal processes through social brain functioning to social behavior.

Keywords: puberty, hormones, adrenarche, gonadarche, brain development, social cognition, social brain

\section{Outline}

Puberty can be defined as the transitional period between childhood and adolescence. It is characterized by substantial change in many areas of development, including hormonal, physical, neuronal, psychological and social. A rapidly expanding body of work shows how development in each of these areas is related to one another. In this chapter, we outline how puberty, and its underlying hormonal and physical changes, might elicit a sensitive period for the development of the social brain. The ultimate aim of puberty is to achieve sexual maturation. However, in humans, a complex set of social skills required to manage adult relationships develops alongside this. We start by giving an 
overview of the hormonal processes underlying puberty and the mechanisms through which they might impact the structure and functioning of the brain. Next, we lay out the knowledge gained so far on how pubertal development relates to the structural and functional development of the social brain. We suggest ways forward to understand the relevance of individual differences in pubertal processes to social brain development, and to unravel the mechanisms from pubertal processes through social brain functioning to social functioning and behavior.

\section{Pubertal processes}

Puberty can generally be divided into two phases. The earliest phase is adrenarche, which starts around age 7-8 (Campbell 2006; Reiter, Fuldauer \& Root 1977). The zona reticularis of the adrenal glands forms during adrenarche, which produces increasing levels of the adrenal hormones dehydroepiandrosterone (DHEA) and its sulphate (DHEAS). The levels of these hormones continue to increase throughout adolescence and reach their peak in early adulthood (Søeborg et al. 2014; Auchus \& Rainey 2004). Both DHEA and DHEAS are neuroactive steroids that can alter neuronal excitability in partially overlapping and partially unique ways (Maninger et al. 2009). They can both be metabolized in the brain and converted into each other. Testosterone also increases during adrenarche, in equivalent levels in boys and girls. During this time, most testosterone is of adrenal origin, metabolized via the conversion of DHEA in the adrenal cortex. DHEA and testosterone show a diurnal pattern with a peak in the early morning (Matchock, Dorn \& Susman 2007). The rise in testosterone during adrenarche is associated with the appearance of some body hair and skin oiliness, though these changes occur later than the hormonal changes and are much more subtle than the physical changes linked to gonadarche.

The more well-known phase of puberty, gonadarche, starts 2-3 years after the start of adrenarche, on average a year earlier in girls than in boys. It is triggered by the re-activation of the hypothalamic-pituitary-gonadal (HPG)-axis, which has been quiescent since infancy. This leads to increases in the levels of luteinizing hormone (LH) and follicle-stimulating hormone (FSH), which later 
on results in steep increases in the levels of the sex hormones testosterone (most strongly for boys) and estradiol and progesterone (most strongly for girls), as the gonads develop (Søeborg et al. 2014). $\mathrm{LH}$ and FSH are released from the pituitary in pulses, mostly during the night, whereas estradiol (like testosterone) shows a diurnal variation that peaks in the morning (Matchock, Dorn \& Susman 2007; Styne \& Grumbach 2011). A monthly cycle in estradiol and progesterone levels develops in girls prior to or around menarche (Hansen, Hoffman \& Ross 1975). Menarche is one of the later steps/milestones in pubertal development for girls and occurs at median age 12.25 years (Biro et al. 2018). The increasing levels of sex hormones trigger the prominent bodily changes (secondary sex characteristics) visible during gonadarche, including breast or testicular growth, and pubic and facial hair. Although most bodily changes occur in early to mid-adolescence, both gonadal and adrenal hormones continue to increase into the third decade of life (Søeborg et al. 2014). The two phases of puberty are suggested to be independent of each other, as children with a clinical disturbance in adrenarche mostly show a normal progression through gonadarche, and vice versa (Styne \& Grumbach 2011).

\section{Measuring puberty}

Several aspects of puberty can be measured and related to other domains of development. To examine normative development, researchers have commonly assessed pubertal status, which refers to the stage of puberty that an individual is currently in. This can be assessed through self-reports or physical examination of secondary sexual characteristics. The most commonly used instruments to measure pubertal stage are the Pubertal Development Scale (Petersen et al. 1988) and the Tanner stage line-drawings (Morris \& Udry 1980), which can both be converted into five stages: pre-, early, mid- late, and post-puberty. Researchers have also indexed normative pubertal development by examining levels of adrenal and gonadal hormones. As discussed in Pubertal processes, levels of these hormones strongly increase during puberty, thus higher hormone levels are considered an indicator of more advanced pubertal development. Part of the studies examining associations between pubertal 
stage or hormone levels and brain development have controlled for age in their models to capture variance uniquely related to pubertal development. The downside is that overlapping variance between age and puberty is filtered out, which is a larger component when the age range is wide. Therefore, in the next sections we mention whether studies controlled for age and specify the age range of study when it is either really narrow or really broad.

Individual variation in when and how rapidly pubertal development occurs, is large. Therefore, other aspects of puberty that can be measured are timing and tempo. Pubertal timing is a person's pubertal status relative to same-age peers, i.e. someone with early timing shows a more advanced development than most of his/her peers. Puberty is considered to be precocious, i.e. clinically early, if gonadarche starts before age 8 for girls or 9 for boys (Latronico, Brito \& Carel 2016). So, above these ages, there is a large normative variation, with ranges for the onset of gonadarche found to be 4 to 5 years in many studies (Biro et al. 2018; Semiz et al. 2008; Aksglaede et al. 2009). Timing of puberty can be measured by taking any of the measures of pubertal status described in the previous paragraph and varying out age, either statistically (taking the residuals from regressing puberty on age) or by taking a group of participants who are all the same age. Further, timing of gonadarche in girls is sometimes conceptualized as the age at menarche.

Finally, tempo refers to the speed of progression through puberty. This also shows substantial variation, for example the time between onset of breast development and age at menarche ranged from 2 to 3.5 years (interquartile range) in a large sample of US girls (Biro et al. 2018). Measuring tempo requires multiple measurements in the same individual (ideally more than two), and this methodological complexity is probably the reason that tempo is the least well-studied aspect of puberty in relation to brain development or health outcomes.

The timing and tempo of adrenarche have gathered much less attention but are thought to also show considerable and relevant variation between children (Barendse et al. 2019; Byrne et al. 2017). The status, timing and tempo of adrenarche and gonadarche can all be examined separately, of 
course depending on the age range studied and the hormonal and physical development indicators collected.

\section{Mechanisms linking puberty and brain development}

Pubertal changes are likely to shape brain structure and functioning through several mechanisms, including a direct impact of hormones on the brain and more indirect pathways, for example through changes in the social environment. The direct impact of hormones on the brain has been studied most extensively in animal research. This has led to an influential theory on the link between hormones and brain structure and function: the organizational-activational hypothesis (adapted to puberty by Schulz, Molenda-Figueira \& Sisk 2009; originally posited by Phoenix et al. 1959). This theory states that steroid hormones influence behavior both through long lasting effects on the organization of neural circuits and through faster activational effects on neural function, although the activational effects can also be long lasting. Although fetal development is the most crucial phase for brain organization, both organizational and activational processes are thought to take place during puberty (Schulz, Molenda-Figueira \& Sisk 2009).

The theory is supported by findings of animal research that testosterone, estradiol, DHEA and DHEAS have effects on neuronal growth, axon growth and survival of neurons (Arevalo, Azcoitia \& Garcia-Segura 2015; Maninger et al. 2009; Ahmed et al. 2008; McCarthy 2008) and can have a long lasting impact on brain organization (Schulz, Molenda-Figueira \& Sisk 2009). These effects are often timing dependent, i.e. administering the hormone to adult animals has no or a different effect. The effects are also regionally specific, depending on (among other things) the distribution of androgen and estrogen receptors. For example, gonadal hormones affect neurogenesis and cellular proliferation in the medial amygdala, leading to bigger volumes in males (De Lorme et al. 2012; Ahmed et al. 2008). Castration of male animals blocks the effects of these hormones, removing the sex difference that normally develops in the volume of the medial amygdala (Ahmed et al. 2008). In the medial prefrontal 
cortex on the other hand, pubertal hormones are thought to influence cell death, also leading to bigger volumes in males (Markham, Morris \& Juraska 2007; Koss et al. 2015). Ovariectomy of female animals prevents this sex difference from occuring, suggesting that it is driven by estrogens or other ovarian hormones.

Apart from organizational effects, adrenal and gonadal hormones can also modulate actions of neurons, having an activational effect. DHEA and DHEAS impact neuronal activation by influencing neurotransmitters, e.g. DHEAS can act as a $\mathrm{GABA}_{A}$ receptor antagonist and both DHEAS and DHEA administration can change the concentration of several neurotransmitters in the hypothalamus (Maninger et al. 2009; Prough, Clark \& Klinge 2016). Testosterone and estradiol do this more indirectly by affecting gene expression after attaching to androgen or estrogen receptors, or by activating other proteins/peptides; estradiol can stimulate the expression of oxytocin for example (Bos et al. 2012). On top of that, adrenal and gonadal hormones can be converted into each other: testosterone is converted to estradiol through aromatization, DHEA and DHEAS can be converted into each other through (de)sulfation, and testosterone is synthesized from DHEA through adrostenedione. It should be noted that most of the mechanisms described above are fully based on animal research and it is unknown how well these proposed mechanisms of action of pubertal hormones on neurobiology translate to humans; even within the animal literature the full extent of these processes is still being determined (Prough, Clark \& Klinge 2016).

\section{The social brain}

The social brain is a broad term to describe a network of regions involved in the processing of social information, understanding the minds of others, and social decision making (Nelson et al. 2005; Burnett et al. 2011; Alcalá-López et al. 2018). The core of this network consists of the temporoparietal junction, superior temporal sulcus, medial prefrontal cortex, and anterior temporal cortex/temporal pole. More broadly, the network encompasses areas for detection of social information, such as from faces; affective or salience areas; regions important for social cognition; and regions involved in 
regulation, integration of information and decision making. The areas for social information detection include the fusiform face area, superior temporal sulcus (STS) and anterior temporal cortex (ATC). Affective areas include the limbic regions, such as the insula and amygdala, as well as the ventral striatum and, in some situations, anterior cingulate cortex (ACC) and medial prefrontal cortex (mPFC). The temporoparietal junction (TPJ), posterior STS, and ATC/temporal pole are involved in social cognition. Regulatory/integration regions include the ACC, mPFC and lateral prefrontal regions. It should be noted that the function of many of these regions is not exclusive to social brain processes, the lateral prefrontal regions for example also have a role in regulating behavior in non-social situations and the ventral striatum is also an indicator of the salience or reward value of non-social stimuli.

A large set of white matter tracts are involved in connecting all of these regions in the social brain. This includes the uncinate fasciculus, connecting amygdala and ventral PFC; the genu of the corpus callosum, connecting left and right PFC; the splenium of the corpus callosum, connecting left with right temporal cortex (and visual cortex); the frontostriatal tracts, connecting the striatum with the PFC; and the cingulum, connecting the ACC to several limbic regions.

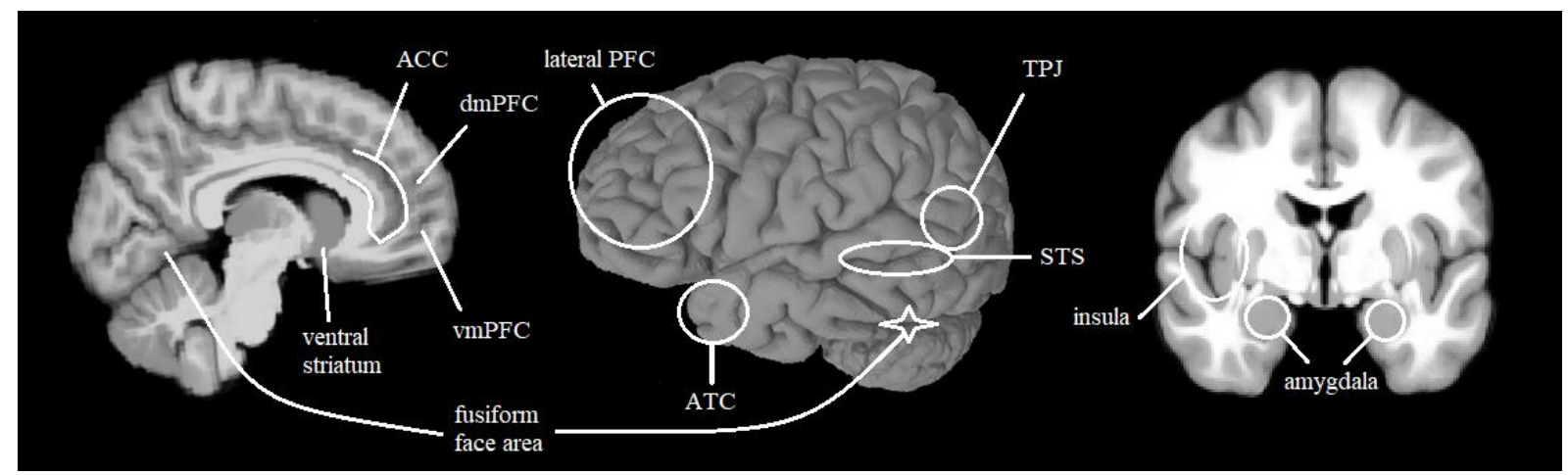

Figure 1. Regions implicated in social brain processes. $A C C=a n t e r i o r$ cingulate cortex, $A T C=a n t e r i o r$ temporal cortex, dmPFC=dorsomedial prefrontal cortex, PFC=prefrontal cortex, STS=superior temporal sulcus, TPJ=temporoparietal junction, vmPFC=ventromedial prefrontal cortex. 


\section{Measuring brain structure and function}

Adolescent brain development is most commonly measured using magnetic resonance imaging (MRI). Different types of MRI scans tap into different aspects of brain development: structural MRI obviously provides information about brain structure, diffusion-weighted imaging (DWI) about white matter structure and connectivity, and functional MRI (fMRI) about brain activation during specific tasks. For more details of the physics behind these different scans, see Weishaupt, Köchli and Marincek (Weishaupt, Köchli \& Marincek 2006).

Structural scans have originally been analyzed to obtain the volumes of cortical and subcortical regions. More recently, researchers have distinguished between cortical thickness and surface area of the cortex, which both underlie cortical volume, but develop in unique ways (Wierenga et al. 2014). Structural MRI scans can additionally be used to measure white matter volume.

DWI is used to obtain more refined properties of the white matter than its overall volume. DWI measures diffusion of water molecules in the brain. In white matter fibres, molecules can easily diffuse along the direction of the fibre, but not in a perpendicular direction. Therefore, much stronger diffusion in one direction compared to other directions indicates the presence of fibres. This diffusion information can be used to extract white matter fiber tracts with tractography, or to create an index of microstructure such as fractional anisotropy (FA), mean diffusivity (MD), radial diffusivity (RD) or axial diffusivity (AD). FA and MD are commonly used, broad summary measures of white matter microstructure, with higher FA indicating stronger diffusion in one direction compared to other directions. $A D$ is diffusivity on the main axis (along the axon), whereas RD is thought of as diffusivity perpendicular to the axon. Recently, more advanced DWI protocols have led to the development of a range of additional indices of white matter (micro)structure, including neurite density index, fiber density, and fiber cross-section. 
Finally, functional MRI provides an indirect index of the activation of brain regions by measuring blood-oxygen-level-dependent (BOLD) signals, based on the fact that active neurons require and receive more oxygen from the blood than inactive neurons. A wide variety of fMRI tasks exists; ones relevant to the social brain include the presentation of affective faces, providing social feedback, excluding the participant during a ball-tossing game, presenting scripts describing social events, or letting the participant reflect on what others think of them. Both functional activation and connectivity can be examined with fMRI, the latter looking at the extent to which brain regions activate 'in synchrony'. In the next sections, we will discuss the current state of the literature on the associations between pubertal processes and each of the aspects of brain development explained above (gray matter structure, white matter structure, and function).

\section{Puberty and brain structure}

Over the course of normal brain development, white matter grows gradually in volume, during adolescence this is likely due to a combination of myelination and increase in axon diameter (Paus 2010). There is also continued development of white matter microstructure, with decreases in MD, and increases in FA, fiber density and fiber cross-section (Genc et al. 2018; Herting et al. 2017). Cortical gray matter however, expands early in life, but reduces in volume during adolescence through cortical thinning and, after early adolescence, reductions in surface area (Lenroot et al. 2007; Mills et al. 2016; Wierenga et al. 2014). The speed and timing of these normal structural changes vary by region of the brain, with basic sensory regions maturing earliest and prefrontal and temporal areas and connected white matter tracts showing much more extended development. Since the social brain contains many frontal and temporal regions, as described in The social brain, it is not surprising that they continue to develop structurally during adolescence. This has been examined in a large longitudinal study by Mills and colleagues (Mills et al. 2014). The findings showed that volume and cortical thickness of the mPFC, TPJ, and posterior STS decrease from late childhood to early adulthood, whereas surface area starts to decrease after early adolescence (around age 13). The ATC showed a different trajectory, with 
increases in cortical thickness during adolescence and decreases in volume and surface area. In the following sections, we will discuss associations between pubertal processes and development of these regions as well as the broader social brain network.

\section{Cortical brain development}

Pubertal development appears to support the normal developmental process of cortical thinning and cortical volume reductions over the course of adolescence. Most studies have reported negative associations between gonadarcheal development (either pubertal stage based on secondary sexual characteristics or gonadal hormone levels) and cortical thickness and volume (Vijayakumar et al. 2018), sometimes dependent on sex. For example, higher testosterone and estradiol levels have been associated with reduced grey matter volume in the ACC and higher testosterone levels with reduced volume in the OFC (Koolschijn, Peper \& Crone 2014). Similarly, a longitudinal study found higher testosterone levels to be associated with lower cortical thickness in the ACC, posterior cingulate and dorsolateral PFC of males (Nguyen, J. McCracken, et al. 2013). Specific to the social brain, increasing pubertal stage has been related to decreasing surface area of the STS in males (Herting et al. 2015); and, in a sample of 9-year-old children, those who had started puberty had decreased mPFC and lateral PFC gray matter density compared to those who were prepubertal (Peper et al. 2009). DHEA seems to have an opposite effect, at least in mid-late childhood, being associated with increasing cortical thickness in the dorsolateral PFC, TPJ, premotor cortex and medial temporal lobe (Nguyen, J. T. McCracken, et al. 2013). In this age range, cortical volumes and thickness are typically still increasing, including in the frontal and temporal lobes (Lenroot et al. 2007), thus DHEA might contribute to these increases.

\section{Subcortical brain development}

Similar to cortical brain development, sex differences have been found in the associations between pubertal development and subcortical brain volumes. The most consistent finding is an association between pubertal development and amygdala volume in males. In male adolescents, 
increasing pubertal development (in the form of secondary sexual characteristics and testosterone levels) has been repeatedly linked with increasing volume of the amygdala (Goddings et al. 2014; Herting et al. 2014; Wierenga et al. 2018; Bramen et al. 2011). The picture is less clear for females, with either weaker increases than males (Goddings et al. 2014), no significant effect (Wierenga et al. 2018), or decreases (Herting et al. 2014; Bramen et al. 2011; Blanton et al. 2012) in amygdala volume with pubertal development.

In the striatum, the findings are also mixed. More developed secondary sexual characteristics (Goddings et al. 2014) and higher testosterone levels (Wierenga et al. 2018) are related to smaller nucleus accumbens in males. In females, no significant association between puberty and nucleus accumbens volume was found in one study (Wierenga et al. 2018) and a negative association in another study (Goddings et al. 2014). Further pubertal development was related to a larger putamen in males but not females (Wierenga et al. 2018). However, another study found a quadratic effect in both sexes: putamen volume did not change or slightly increased from prepuberty to mid-puberty, but then decreased from mid- to post-puberty (Goddings et al. 2014).

For the hippocampus, effects of pubertal development also seem to be sex-specific. Negative associations have been found between pubertal development based on secondary sexual characteristics and hippocampus volume in females (Bramen et al. 2011; Blanton et al. 2012), and positive associations with secondary sexual characteristics or testosterone in males (Bramen et al. 2011; Goddings et al. 2014; Wierenga et al. 2018). However, one study found no significant associations between secondary sexual characteristics or testosterone levels and hippocampal volumes (Herting et al. 2014).

Associations between pubertal development and brain volume have also been found in other subcortical regions, such as the thalamus and globus pallidus, but again with inconsistent results (Goddings et al. 2014; Wierenga et al. 2018). Finally, positive associations between pubertal 
development and pituitary volume have been reported, as well as larger volumes in those with early timing (Murray et al. 2016; Peper et al. 2010; Whittle et al. 2012; Wong et al. 2014).

\section{White matter development}

Levels of pubertal hormones as well as secondary sexual characteristics are associated with measures of brain connectivity (Perrin et al. 2008; Herting et al. 2012; Peper et al. 2008; Menzies et al. 2015; Herting et al. 2017). Most of these associations have been found after controlling for age. More advanced pubertal stage appeared to be related to larger volumes of white matter, but findings are more mixed for microstructure.

Several studies have examined white matter microstructure in relation to secondary sexual characteristics (Asato et al. 2010; Menzies et al. 2015; Herting et al. 2017; Genc et al. 2017). In the first study, with a wide age range (8-28 years old), those with more developed secondary sexual characteristics had more developed white matter microstructure (Asato et al. 2010). Almost all investigated tracts continued to develop throughout the whole course of puberty, including tracts relevant to the social brain like the uncinate fasciculus, frontostriatal connections, and the corpus callosum. In line with this, two more recent studies have demonstrated lower mean diffusivity (MD) across many major tracts (Menzies et al. 2015) and higher fibre density in the corpus callosum (Genc et al. 2017) in participants with more advanced pubertal maturation. However, the two longitudinal studies on changes in secondary sexual characteristics in relation to white matter microstructure development have shown a more complex pattern, with no associations with change in fibre density and sex-specific (both positive and negative) associations with change in fractional anisotropy (FA) (Herting et al. 2017; Genc et al. 2018).

Studies including sex hormone levels have shown varied associations with white matter microstructure. This includes associations in the direction of normal white matter development, such as a positive association between morning serum testosterone and whole-brain white matter volume (controlling for age; Perrin et al. 2008), and positive relations of morning serum testosterone and 
estradiol levels, controlling for age, with FA values in many white matter tracts (Herting et al. 2012). Also, negative associations of maturation of secondary sexual characteristics and testosterone levels with MD values have been found in many white matter tracts in males, including tracts connecting social brain areas such as the uncinate fasciculus, cingulum and corpus callosum (Menzies et al. 2015). However, there are also some opposite and null findings. For example, one study in a sample of 8- to 25-year-olds found a positive association between morning saliva testosterone levels and MD, most strongly in females, and no significant results for estradiol (Peper et al. 2015). A study in 9-year-old children showed a positive association between morning saliva DHEA levels and MD in a wide range of tracts (Barendse et al. 2018). Since the study included a very limited age range and controlled for age, this finding was interpreted as an effect of relatively early timing of adrenarche. No studies to date have examined longitudinal associations between pubertal hormones and white matter microstructure.

\section{Summary}

The research so far indicates that effects of secondary sexual characteristics and of adrenal and gonadal hormones on white matter development might be widespread. Effects on cortical and subcortical brain development appear less widespread and less consistent across the brain. They still include much of the social brain, however, such as the medial and lateral PFC, ACC, STS, and amygdala. The mechanisms through which pubertal hormones can influence brain structure might differ between the gray and white matter because of different receptor distributions or distributions of glial cells. Androgen and estrogen receptors have been found in several types of glia, including astrocytes and oligodendrocytes (Finley \& Kritzer 1999; Jung-Testas \& Baulieu 1998), which have different functions in the gray and white matter. Besides that, actions of pubertal hormones on cell bodies and dendrites (i.e. gray matter) can have downstream effects on the connected white matter. Pubertal influences are further likely to vary between gray matter regions because of varying levels of androgen and estrogen receptors. 
However, findings are also not completely consistent within regions/tracts, with some studies reporting effects in opposite directions compared to other studies. As illustrated by the findings reviewed above, associations between hormone levels and subcortical and cortical brain volumes depend on sex. Findings might have additionally differed between studies because of the varying age ranges studied and analysis techniques applied. Animal research has shown that mechanisms of gonadal hormone action can differ between subregions of subcortical areas (De Lorme et al. 2012; Meyer, Ferres-Torres \& Mas 1978), which could also contribute to inconsistencies in how levels of these hormones relate to volume of the whole area. Further longitudinal research in large samples is needed, as well as direct comparisons of analysis techniques and analysis of subregions, to shed light on the source of inconsistencies in the current literature.

\section{Puberty and social brain function}

A popular idea in the field of adolescent brain development is that puberty renders adolescents more sensitive to social information by altering the way in which their brain processes this information (Nelson et al. 2005). This could in turn affect their decision making during social situations. Recent research has provided some support for this hypothesis, using a range of functional MRI paradigms, including viewing affective faces, rejection/exclusion, and social cognition paradigms.

\section{Affective face processing}

Facial expressions are a prevalent source of social information and therefore presenting affective faces is a commonly used paradigm in research on socio-affective brain function. Several studies have examined associations between pubertal development and neural correlates of affective face processing, often focusing on the amygdala because of its well-known role in emotion processing. Two studies using ROI analyses reported decreased amygdala activation to neutral faces in those with more developed secondary sexual characteristics, but no difference in amygdala activation to fearful faces (Ferri et al. 2014; Forbes et al. 2011). This suggests that pubertal development is related to decreased salience of neutral faces, rather than increased salience of affective faces. The two 
longitudinal study on this topic, however, have shown inconsistent results. Spielberg and colleagues (2014) reported that increasing testosterone levels across early adolescence were positively linked to increasing amygdala reactivity to angry and fearful faces, with a follow-up study in the same sample showing an additional link with reduced connectivity between the amygdala and the orbitofrontal cortex (Spielberg et al. 2015). Vijayakumar and colleagues (2019) on the other hand, showed a quadratic (U-shaped) association between testosterone and amygdala activation in females. Further, Spielberg and colleagues (2014) found positive associations between nucleus accumbens activity and changes in testosterone levels, whereas Vijayakumar and colleagues (2019) reported no association with testosterone levels and a negative association with secondary sexual characteristics. These discrepancies might be partially due to the wider age range in Vijayakumar and colleagues (2019) and partly to the fact that Spielberg and colleagues (2014) focused on the amount of intraindividual change in hormone level and activation. The findings also show that hormonal changes (or specifically changes in testosterone) might have different effects than pubertal development as indexed by physical characteristics. Finally, a study on pubertal timing reported increased amygdala, visual cortex and thalamus activation to both neutral and affective faces in those with earlier pubertal timing (at age 10 and age 13; Moore et al. 2012), suggesting individual variation in pubertal development is relevant to affective face processing.

The studies above have all focused on gonadarcheal development. Adrenarcheal development has also been linked to neural activation to affective faces; higher DHEA levels, controlling for age, have been related to decreased midcingulate activation to affective compared to calm faces (but not relation with amygdala activation; Whittle et al. 2015). The authors interpreted this as timing of adrenarche moderating affective face processing.

\section{Social feedback and ostracism}

Another common way to examine sensitivity to social information is to provide participants with peer feedback, in the form of acceptance and rejection or inclusion and exclusion. Being included 
or accepted elicits activation in reward-related areas of the striatum and in the medial prefrontal cortex (Dalgleish et al. 2017; Sherman et al. 2016). Rejection and exclusion on the other hand activate the subgenual anterior cingulate cortex and precuneus/posterior cingulate, as well as the regulatory region of the ventrolateral prefrontal cortex (Vijayakumar, Cheng \& Pfeifer 2017). Surprisingly, only one study has examined the association between pubertal development and neural activation during peer rejection. In this study, having more developed secondary sexual characteristics was related to more activation in the bilateral amygdala/parahippocampal gyrus and the caudate/subgenual anterior cingulate in response to rejection by peers (Silk et al. 2014). These effects remained significant when controlling for age. From this, it seems that part of the normal neural responses to rejection become strengthened during puberty. The neural response to acceptance feedback, however, was not associated with pubertal development. Replication of this finding is warranted, particularly using social feedback paradigms that reflect (online or offline) social interactions that adolescents have in their everyday life (e.g. Sherman et al. 2016).

\section{Social cognition}

More advanced social-cognitive skills, including mentalizing and perspective taking, are still developing in adolescence (Dumontheil, Apperly \& Blakemore 2010). Several studies have examined how neural processes underlying these skills develop and relate to puberty. In a small sample of early adolescents $(\mathrm{N}=16)$, more developed secondary sexual characteristics at age 13 , and greater longitudinal increases in development of these characteristics from age 10 to 13 , related to increased activity in social cognition related regions while witnessing peer rejection (including dorsomedial PFC, posterior cingulate, TPJ and temporal pole) (Masten et al. 2013). This suggests increasing engagement of social brain areas with pubertal development during a perspective taking/empathy task. Also, activity in many of these same brain areas was related to higher self-reported empathic abilities, and increasing empathic abilities over time, suggesting that these areas may play a role in the development of social-cognitive skills. Building on studies focused on basic emotion processing, another study looked at associations between puberty and processing of social emotions (embarrassment and guilt), 
which require more perspective taking than basic emotions (Goddings et al. 2012). This study (in girls only) showed that adolescents with higher testosterone levels, controlled for age, had increased temporal pole activation while processing social emotions. However, there were no differences in activation between those at lower versus higher pubertal stage based on secondary sexual characteristics.

\section{Self-perception}

Another set of studies have begun investigating how puberty relates to the neural correlates of self-evaluation. Although thinking about oneself is not a social behavior in itself, people extensively use social information to inform their self-evaluations. One study looked explicitly at this by using reflected self-evaluations (evaluating oneself in the eyes of a peer) and demonstrated increased activation in a ventral striatum ROI for those with more developed secondary sexual characteristics (although in a small sample [ $\mathrm{N}=18]$, (Jankowski et al. 2014)). The first such study examining neural activation in relation to direct self-evaluation observed that greater within-individual change in selfreported pubertal development over three years was associated with larger increases in vmPFC activation during self-evaluation of social traits in particular (Pfeifer et al. 2013). These preliminary investigations led to a subsequent cross-sectional investigation of 143 early adolescent girls, ages 1013 years, who completed a similar self-evaluation fMRI task and also provided data to compute several metrics of pubertal development (self-report and basal salivary hormone levels over the previous month). Contrary to expectations, more advanced pubertal development was not associated with greater activity in anterior cortical midline structures (defined a priori using a parcellation map) during self-evaluation of social traits (Barendse et al. 2019), despite having $95 \%$ power to detect an $\mathrm{f}^{2}$ of 0.12 or larger. It is possible that pubertal effects are very small in cross-sectional data during early adolescence, are most evident in association with longitudinal changes within individuals, or are sensitive to the domains of self-concept assessed and contrasted with each other. For example, a large fMRI study of self-evaluations across social, academic, and physical appearance domains did not report 
pubertal associations, but did find that MPFC activity increased with age for physical appearance selfevaluations only (van der Cruijsen et al. 2018).

\section{Summary}

Pubertal development has been associated with the neural processing of various forms of social information. The salience of neutral facial expressions might be lower at more advanced pubertal stages, but the evidence is mixed on whether (negative) affective faces are more salient for those at more advanced pubertal development. Further, pubertal development might increase adolescents' sensitivity to rejection feedback (in terms of higher amygdala and subgenual anterior cingulate activation), but not to acceptance. Pubertal development is also linked to increased engagement of the social brain during social cognition tasks. This might support further development of social-cognitive skills (Masten et al. 2013), although not many studies have directly tested associations with social behavior.

However, the strength of all of these conclusions is limited by the small sample sizes used, the lack of replications and the low number of longitudinal studies. Also, there is limited research on individual variation in the timing or tempo of puberty in relation to social brain development, even though this variation is large and has been consistently linked to mental health outcomes (Ullsperger \& Nikolas 2017).

\section{Future directions and conclusion}

Overall, both the animal and human literature to date suggests that pubertal development is relevant for the development of both structure and function of the social brain. Pubertal status has been associated with the structural development of many social brain regions and their connections, partly in interaction with sex. Functionally, puberty might increase the sensitivity of the brain to social information. Pubertal timing, although based on a much more limited set of studies, has also been 
associated with brain structure and function in adolescence. However, conclusions on the direction and localization of associations between puberty and brain development is limited by a range of methodological issues that have led to inconsistencies between studies. These issues include small sample sizes, variation in the age range of studies, in the measures of pubertal development and in the techniques used to analyze brain structure. To determine the origin of the inconsistencies and resolve them, larger longitudinal projects are needed as well as studies that apply and compare multiple analysis techniques for structural brain development.

Studies that examined pubertal development using both hormone levels and self-report of physical changes (secondary sexual characteristics) have often found differential effects of both. It remains important to examine both of these indicators of pubertal development, especially when focusing on the social brain. This is because only physical changes directly impact how other people perceive you and how you perceive yourself, which in turn can influence social interactions that shape the social brain. This more indirect, psychosocial mechanism is in contrast to the direct mechanisms that are often suggested of hormonal impact on brain development and lacks empirical investigation even though it has been suggested as a mechanism linking pubertal processes to risk for e.g. depression in girls (Cyranowski et al. 2000; Mendle, Turkheimer \& Emery 2007).

A related point is that studies on the links between pubertal development and social brain function have often neglected to examine actual social behavior or functioning. This is an important avenue for future research because examining mechanisms from (variation in) pubertal development through brain development to social functioning will help us understand the origins of changes in social behavior of adolescents.

Further, as described in Measuring puberty, interindividual differences in the timing and tempo of pubertal processes are large. These differences are also relevant for mental health (Barendse et al. 2019; Belsky et al. 2015; Ullsperger \& Nikolas 2017). However, most of the research to date examined pubertal stage and thus normal developmental changes. One of the reasons might be that 
it is complex to acquire a dataset with enough participants and time points to examine variation in change over time between individuals, especially when including measures of brain development. Nonetheless, it is important for future research to examine this variation because of the relevance for identifying adolescents at risk for mental illness or dysfunctional behavior and eventually creating more targeted prevention or intervention efforts.

In conclusion, there is evidence demonstrating that pubertal development is relevant for the development of both structure and function of the social brain. However, methodological issues such as small sample size, and variation in study design and analysis, limit the possibility of drawing more specific conclusions. Apart from overcoming these methodological problems, future research is needed that focuses on individual differences in pubertal processes and their relevance to development of the social brain, as well as research examining the mechanisms from pubertal processes through social brain functioning to social behavior.

\section{References}

Ahmed, El, Zehr, JL, Schulz, KM, Lorenz, BH, DonCarlos, LL \& Sisk, CL 2008, 'Pubertal hormones modulate the addition of new cells to sexually dimorphic brain regions', Nature Neuroscience, vol. 11, no. 9, pp. 995-997.

Aksglaede, L, Sorensen, K, Petersen, JH, Skakkebaek, NE \& Juul, A 2009, 'Recent Decline in Age at Breast Development: The Copenhagen Puberty Study', Pediatrics, vol. 123, no. 5, pp. e932e939.

Alcalá-López, D, Smallwood, J, Jefferies, E, Van Overwalle, F, Vogeley, K, Mars, RB, Turetsky, BI, Laird, AR, Fox, PT, Eickhoff, SB \& Bzdok, D 2018, 'Computing the Social Brain Connectome Across Systems and States', Cerebral Cortex, vol. 28, no. 7, pp. 2207-2232.

Arevalo, M-A, Azcoitia, I \& Garcia-Segura, LM 2015, 'The neuroprotective actions of oestradiol and oestrogen receptors.', Nature reviews. Neuroscience, vol. 16, no. 1, pp. 17-29.

Asato, MR, Terwilliger, R, Woo, J \& Luna, B 2010, 'White matter development in adolescence: a DTI study.', Cerebral cortex, vol. 20, no. 9, pp. 2122-31.

Auchus, RJ \& Rainey, WE 2004, 'Adrenarche - physiology, biochemistry and human disease', Clinical Endocrinology, vol. 60, no. 3, pp. 288-296.

Barendse, MEA, Cosme, D, Flournoy, JC, Vijayakumar, N, Cheng, TW, Allen, NB, Pfeifer, JH 2019, Neural correlates of self-evaluation during puberty [WWW Document]. 
doi:10.31234/osf.io/r2pjm

Barendse, MEA, Simmons, JG, Byrne, ML, Seal, ML, Patton, G, Mundy, L, Wood, SJ, Olsson, CA, Allen, NB \& Whittle, S 2018, 'Brain structural connectivity during adrenarche: Associations between hormone levels and white matter microstructure.', Psychoneuroendocrinology, vol. 88, pp. 7077.

Barendse, MEA, Simmons, JG, Patton, G, Mundy, L, Byrne, ML, Seal, ML, Allen, NB \& Whittle, S 2019, 'Adrenarcheal Timing Longitudinally Predicts Anxiety Symptoms Via Amygdala Connectivity During Emotion Processing', Journal of the American Academy of Child \& Adolescent Psychiatry.

Belsky, J, Ruttle, PL, Boyce, WT, Armstrong, JM \& Essex, MJ 2015, 'Early adversity, elevated stress physiology, accelerated sexual maturation, and poor health in females.', Developmental psychology, vol. 51, no. 6, pp. 816-22.

Biro, FM, Pajak, A, Wolff, MS, Pinney, SM, Windham, GC, Galvez, MP, Greenspan, LC, Kushi, LH \& Teitelbaum, SL 2018, 'Age of Menarche in a Longitudinal US Cohort.', Journal of pediatric and adolescent gynecology, vol. 31, no. 4, pp. 339-345.

Blanton, RE, Cooney, RE, Joormann, J, Eugène, F, Glover, GH \& Gotlib, IH 2012, 'Pubertal stage and brain anatomy in girls.', Neuroscience, vol. 217, pp. 105-12.

Bos, P a., Panksepp, J, Bluthé, RM \& Honk, J Van 2012, 'Acute effects of steroid hormones and neuropeptides on human social-emotional behavior: A review of single administration studies', Frontiers in Neuroendocrinology, vol. 33, no. 1, pp. 17-35.

Bramen, JE, Hranilovich, JA, Dahl, RE, Forbes, EE, Chen, J, Toga, AW, Dinov, ID, Worthman, CM \& Sowell, ER 2011, 'Puberty influences medial temporal lobe and cortical gray matter maturation differently in boys than girls matched for sexual maturity.', Cerebral cortex, vol. 21, no. 3, pp. 636-46.

Burnett, S, Sebastian, C, Cohen Kadosh, K \& Blakemore, S-J 2011, 'The social brain in adolescence: evidence from functional magnetic resonance imaging and behavioural studies.', Neuroscience and biobehavioral reviews, vol. 35, no. 8, pp. 1654-64.

Byrne, ML, Whittle, S, Vijayakumar, N, Dennison, M, Simmons, JG \& Allen, NB 2017, 'A systematic review of adrenarche as a sensitive period in neurobiological development and mental health', Developmental Cognitive Neuroscience, vol. 25, pp. 12-28.

Campbell, B 2006, 'Adrenarche and the evolution of human life history.', American journal of human biology : the official journal of the Human Biology Council, vol. 18, no. 5, pp. 569-89.

van der Cruijsen, R, Peters, S, van der Aar, LPE \& Crone, EA 2018, 'The neural signature of selfconcept development in adolescence: The role of domain and valence distinctions', Developmental Cognitive Neuroscience, vol. 30, pp. 1-12.

Cyranowski, JM, Frank, E, Young, E \& Shear, MK 2000, 'Adolescent onset of the gender difference in lifetime rates of major depression: a theoretical model.', Archives of general psychiatry, vol. 57, no. 1 , pp. 21-7.

Dalgleish, T, Walsh, ND, Mobbs, D, Schweizer, S, van Harmelen, A-L, Dunn, B, Dunn, V, Goodyer, I \& Stretton, J 2017, 'Social pain and social gain in the adolescent brain: A common neural circuitry underlying both positive and negative social evaluation', Scientific Reports, vol. 7, no. 1, p. 42010.

Dumontheil, I, Apperly, IA \& Blakemore, S-J 2010, 'Online usage of theory of mind continues to develop in late adolescence', Developmental Science, vol. 13, no. 2, pp. 331-338. 
Ferri, J, Bress, JN, Eaton, NR \& Proudfit, GH 2014, 'The Impact of Puberty and Social Anxiety on Amygdala Activation to Faces in Adolescence', Developmental Neuroscience, vol. 36, no. 3-4, pp. 239-249.

Finley, SK \& Kritzer, MF 1999, 'Immunoreactivity for intracellular androgen receptors in identified subpopulations of neurons, astrocytes and oligodendrocytes in primate prefrontal cortex', Journal of Neurobiology, vol. 40, no. 4, pp. 446-457.

Forbes, EE, Phillips, ML, Silk, JS, Ryan, ND \& Dahl, RE 2011, 'Neural Systems of Threat Processing in Adolescents: Role of Pubertal Maturation and Relation to Measures of Negative Affect', Developmental Neuropsychology, vol. 36, no. 4, pp. 429-452.

Genc, S, Seal, ML, Dhollander, T, Malpas, CB, Hazell, P \& Silk, TJ 2017, 'White matter alterations at pubertal onset', Neurolmage, vol. 156, pp. 286-292.

Genc, S, Smith, RE, Malpas, CB, Anderson, V, Nicholson, JM, Efron, D, Sciberras, E, Seal, ML \& Silk, TJ 2018, 'Development of white matter fibre density and morphology over childhood: A longitudinal fixel-based analysis', Neurolmage, vol. 183, pp. 666-676.

Goddings, A-L, Burnett Heyes, S, Bird, G, Viner, RM \& Blakemore, S-J 2012, 'The relationship between puberty and social emotion processing.', Developmental science, vol. 15, no. 6, pp. 801-11.

Goddings, A-L, Mills, KL, Clasen, LS, Giedd, JN, Viner, RM \& Blakemore, S-J 2014, 'The influence of puberty on subcortical brain development.', Neurolmage, vol. 88, pp. 242-51.

Hansen, JW, Hoffman, HJ \& Ross, GT 1975, 'Monthly gonadotropin cycles in premenarcheal girls.', Science, vol. 190, no. 4210, pp. 161-3.

Herting, MM, Gautam, P, Spielberg, JM, Dahl, RE \& Sowell, ER 2015, 'A longitudinal study: changes in cortical thickness and surface area during pubertal maturation.', PloS one, vol. 10, no. 3, p. e0119774.

Herting, MM, Gautam, P, Spielberg, JM, Kan, E, Dahl, RE \& Sowell, ER 2014, 'The role of testosterone and estradiol in brain volume changes across adolescence: a longitudinal structural MRI study.', Human brain mapping, vol. 35, no. 11, pp. 5633-45.

Herting, MM, Kim, R, Uban, KA, Kan, E, Binley, A \& Sowell, ER 2017, 'Longitudinal changes in pubertal maturation and white matter microstructure', Psychoneuroendocrinology, vol. 81, pp. 70-79.

Herting, MM, Maxwell, EC, Irvine, C \& Nagel, BJ 2012, 'The Impact of Sex, Puberty, and Hormones on White Matter Microstructure in Adolescents', Cerebral Cortex, vol. 22, no. 9, pp. 1979-1992.

Jankowski, KF, Moore, WE, Merchant, JS, Kahn, LE \& Pfeifer, JH 2014, 'But do you think I'm cool?: Developmental differences in striatal recruitment during direct and reflected social selfevaluations', Developmental Cognitive Neuroscience, vol. 8, pp. 40-54.

Jung-Testas, I \& Baulieu, EE 1998, 'Steroid hormone receptors and steroid action in rat glial cells of the central and peripheral nervous system', The Journal of Steroid Biochemistry and Molecular Biology, vol. 65, no. 1-6, pp. 243-251.

Koolschijn, PCMP, Peper, JS \& Crone, EA 2014, 'The influence of sex steroids on structural brain maturation in adolescence.', PloS one, vol. 9, no. 1, p. e83929.

Koss, WA, Lloyd, MM, Sadowski, RN, Wise, LM \& Juraska, JM 2015, 'Gonadectomy before puberty increases the number of neurons and glia in the medial prefrontal cortex of female, but not male, rats', Developmental Psychobiology, vol. 57, no. 3, pp. 305-312.

Latronico, AC, Brito, VN \& Carel, J-C 2016, 'Causes, diagnosis, and treatment of central precocious 
puberty', The Lancet Diabetes \& Endocrinology, vol. 4, no. 3, pp. 265-274.

Lenroot, RK, Gogtay, N, Greenstein, DK, Wells, EM, Wallace, GL, Clasen, LS, Blumenthal, JD, Lerch, J, Zijdenbos, AP, Evans, AC, Thompson, PM \& Giedd, JN 2007, 'Sexual dimorphism of brain developmental trajectories during childhood and adolescence.', Neurolmage, vol. 36, no. 4, pp. 1065-73.

De Lorme, KC, Schulz, KM, Salas-Ramirez, KY \& Sisk, CL 2012, 'Pubertal testosterone organizes regional volume and neuronal number within the medial amygdala of adult male Syrian hamsters', Brain Research, vol. 1460, pp. 33-40.

Maninger, N, Wolkowitz, OM, Reus, VI, Epel, ES \& Mellon, SH 2009, 'Neurobiological and neuropsychiatric effects of dehydroepiandrosterone (DHEA) and DHEA sulfate (DHEAS).', Frontiers in neuroendocrinology, vol. 30, no. 1, pp. 65-91.

Markham, JA, Morris, JR \& Juraska, JM 2007, 'Neuron number decreases in the rat ventral, but not dorsal, medial prefrontal cortex between adolescence and adulthood', Neuroscience, vol. 144, no. 3, pp. 961-968.

Masten, CL, Eisenberger, NI, Pfeifer, JH, Colich, NL \& Dapretto, M 2013, 'Associations among pubertal development, empathic ability, and neural responses while witnessing peer rejection in adolescence', Child Development, vol. 84, no. 4, pp. 1338-1354.

Matchock, RL, Dorn, LD \& Susman, EJ 2007, 'Diurnal and seasonal cortisol, testosterone, and DHEA rhythms in boys and girls during puberty.', Chronobiology international, vol. 24, no. 5, pp. 969990.

McCarthy, MM 2008, 'Estradiol and the developing brain.', Physiological reviews, vol. 88, no. 1, pp. 91-124.

Mendle, J, Turkheimer, E \& Emery, RE 2007, 'Detrimental psychological outcomes associated with early pubertal timing in adolescent girls', Developmental Review, vol. 27, no. 2, pp. 151-171.

Menzies, L, Goddings, A-L, Whitaker, KJ, Blakemore, S-J \& Viner, RM 2015, 'The effects of puberty on white matter development in boys.', Developmental cognitive neuroscience, vol. 11, pp. 116-28.

Meyer, G, Ferres-Torres, R \& Mas, M 1978, 'The effects of puberty and castration on hippocampal dendritic spines of mice. A Golgi study', Brain Research, vol. 155, no. 1, pp. 108-112.

Mills, KL, Goddings, A-L, Herting, MM, Meuwese, R, Blakemore, S-J, Crone, EA, Dahl, RE, Güroğlu, B, Raznahan, A, Sowell, ER \& Tamnes, CK 2016, 'Structural brain development between childhood and adulthood: Convergence across four longitudinal samples', Neurolmage, vol. 141, pp. 273281.

Mills, KL, Lalonde, F, Clasen, LS, Giedd, JN \& Blakemore, S-J 2014, 'Developmental changes in the structure of the social brain in late childhood and adolescence.', Social cognitive and affective neuroscience, vol. 9, no. 1, pp. 123-31.

Moore, WE, Pfeifer, JH, Masten, CL, Mazziotta, JC, lacoboni, M \& Dapretto, M 2012, 'Facing puberty: associations between pubertal development and neural responses to affective facial displays.', Social cognitive and affective neuroscience, vol. 7, no. 1, pp. 35-43.

Morris, NM \& Udry, JR 1980, 'Validation of a self-administered instrument to assess stage of adolescent development', Journal of Youth and Adolescence, vol. 9, no. 3, pp. 271-280.

Murray, CR, Simmons, JG, Allen, NB, Byrne, ML, Mundy, LK, Seal, ML, Patton, GC, Olsson, CA \& Whittle, S 2016, 'Associations between dehydroepiandrosterone (DHEA) levels, pituitary volume, and social anxiety in children', Psychoneuroendocrinology, vol. 64, pp. 31-39. 
Nelson, EE, Leibenluft, E, McClure, EB \& Pine, DS 2005, 'The social re-orientation of adolescence: a neuroscience perspective on the process and its relation to psychopathology', Psychological Medicine, vol. 35, no. 2, pp. 163-174.

Nguyen, T-V, McCracken, J, Ducharme, S, Botteron, KN, Mahabir, M, Johnson, W, Israel, M, Evans, AC \& Karama, S 2013, 'Testosterone-related cortical maturation across childhood and adolescence.', Cerebral cortex, vol. 23, no. 6, pp. 1424-32.

Nguyen, T-V, McCracken, JT, Ducharme, S, Cropp, BF, Botteron, KN, Evans, AC \& Karama, S 2013, 'Interactive effects of dehydroepiandrosterone and testosterone on cortical thickness during early brain development.', The Journal of neuroscience, vol. 33, no. 26, pp. 10840-8.

Paus, T 2010, 'Growth of white matter in the adolescent brain: myelin or axon?', Brain and cognition, vol. 72 , no. 1 , pp. 26-35.

Peper, JS, Brouwer, RM, van Leeuwen, M, Schnack, HG, Boomsma, DI, Kahn, RS \& Hulshoff Pol, HE 2010, 'HPG-axis hormones during puberty: a study on the association with hypothalamic and pituitary volumes.', Psychoneuroendocrinology, vol. 35, no. 1, pp. 133-40.

Peper, JS, Brouwer, RM, Schnack, HG, van Baal, GCM, van Leeuwen, M, van den Berg, SM, DelemarreVan de Waal, HA, Janke, AL, Collins, DL, Evans, AC, Boomsma, DI, Kahn, RS \& Hulshoff Pol, HE 2008, 'Cerebral white matter in early puberty is associated with luteinizing hormone concentrations.', Psychoneuroendocrinology, vol. 33, no. 7, pp. 909-15.

Peper, JS, de Reus, MA, van den Heuvel, MP \& Schutter, DJLG 2015, 'Short fused? associations between white matter connections, sex steroids, and aggression across adolescence.', Human brain mapping, vol. 36, no. 3, pp. 1043-52.

Peper, JS, Schnack, HG, Brouwer, RM, Van Baal, GCM, Pjetri, E, Székely, E, van Leeuwen, M, van den Berg, SM, Collins, DL, Evans, AC, Boomsma, DI, Kahn, RS \& Hulshoff Pol, HE 2009, 'Heritability of regional and global brain structure at the onset of puberty: a magnetic resonance imaging study in 9-year-old twin pairs.', Human brain mapping, vol. 30, no. 7, pp. 2184-96.

Perrin, JS, Hervé, P-Y, Leonard, G, Perron, M, Pike, GB, Pitiot, A, Richer, L, Veillette, S, Pausova, Z \& Paus, T 2008, 'Growth of white matter in the adolescent brain: role of testosterone and androgen receptor.', The Journal of Neuroscience, vol. 28, no. 38, pp. 9519-24.

Petersen, AC, Crockett, L, Richards, M \& Boxer, A 1988, 'A self-report measure of pubertal status: Reliability, validity, and initial norms', Journal of Youth and Adolescence, vol. 17, no. 2, pp. 117133.

Pfeifer, JH, Kahn, LE, Merchant, JS, Peake, SJ, Veroude, K, Masten, CL, Lieberman, MD, Mazziotta, JC \& Dapretto, M 2013, 'Longitudinal change in the neural bases of adolescent social selfevaluations: effects of age and pubertal development.', The Journal of neuroscience, vol. 33, no. 17, pp. 7415-9.

Phoenix, CH, Goy, RW, Gerall, AA \& Young, WC 1959, 'Organizing action of prenatally administered testosterone propionate on the tissues mediating mating behavior in the female guinea pig.', Endocrinology, vol. 65, pp. 369-82.

Prough, RA, Clark, BJ \& Klinge, CM 2016, 'Novel mechanisms for DHEA action', Journal of Molecular Endocrinology.

Reiter, EO, Fuldauer, VG \& Root, AW 1977, 'Secretion of the adrenal androgen, dehydroepiandrosterone sulfate, during normal infancy, childhood, and adolescence, in sick infants, and in children with endocrinologic abnormalities', The Journal of Pediatrics, vol. 90, no. 5, pp. 766-770. 
Schulz, KM, Molenda-Figueira, HA \& Sisk, CL 2009, 'Back to the future: The organizational-activational hypothesis adapted to puberty and adolescence.', Hormones and behavior, vol. 55, no. 5, pp. 597-604.

Semiz, S, Kurt, F, Kurt, DT, Zencir, M \& Sevinç, O 2008, 'Pubertal development of Turkish children.', Journal of pediatric endocrinology \& metabolism : JPEM, vol. 21, no. 10, pp. 951-61.

Sherman, LE, Payton, AA, Hernandez, LM, Greenfield, PM \& Dapretto, M 2016, 'The Power of the Like in Adolescence: Effects of Peer Influence on Neural and Behavioral Responses to Social Media.', Psychological science, vol. 27, no. 7, pp. 1027-35.

Silk, JS, Siegle, GJ, Lee, KH, Nelson, EE, Stroud, LR \& Dahl, RE 2014, 'Increased neural response to peer rejection associated with adolescent depression and pubertal development.', Social cognitive and affective neuroscience, vol. 9, no. 11, pp. 1798-807.

Søeborg, T, Frederiksen, H, Mouritsen, A, Johannsen, TH, Main, KM, Jørgensen, N, Petersen, JH, Andersson, A-M \& Juul, A 2014, 'Sex, age, pubertal development and use of oral contraceptives in relation to serum concentrations of DHEA, DHEAS, $17 \alpha$-hydroxyprogesterone, $\Delta 4$ androstenedione, testosterone and their ratios in children, adolescents and young adults.', Clinica chimica acta; international journal of clinical chemistry, vol. 437, pp. 6-13.

Spielberg, JM, Forbes, EE, Ladouceur, CD, Worthman, CM, Olino, TM, Ryan, ND \& Dahl, RE 2015, 'Pubertal testosterone influences threat-related amygdala-orbitofrontal cortex coupling.', Social cognitive and affective neuroscience, vol. 10, no. 3, pp. 408-15.

Spielberg, JM, Olino, TM, Forbes, EE \& Dahl, RE 2014, 'Exciting fear in adolescence: does pubertal development alter threat processing?', Developmental cognitive neuroscience, vol. 8, pp. 86-95.

Styne, DM \& Grumbach, MM 2011, 'Puberty: Ontogeny, Neuroendocrinology, Physiology, and Disorders', in S Melmed, KS Polonsky, PR Larsen, \& HM Kronenberg (eds), Williams Textbook of Endocrinology, Elsevier, Philadelphia, pp. 1054-1201.

Ullsperger, JM \& Nikolas, MA 2017, 'A meta-analytic review of the association between pubertal timing and psychopathology in adolescence: Are there sex differences in risk?', Psychological Bulletin, vol. 143, no. 9, pp. 903-938.

Vijayakumar, N, Cheng, TW \& Pfeifer, JH 2017, 'Neural correlates of social exclusion across ages: A coordinate-based meta-analysis of functional MRI studies', Neurolmage, vol. 153, pp. 359-368.

Vijayakumar, N, Op de Macks, Z, Shirtcliff, EA \& Pfeifer, JH 2018, 'Puberty and the human brain: Insights into adolescent development', Neuroscience \& Biobehavioral Reviews, vol. 92, pp. 417436.

Vijayakumar, N, Pfeifer, JH, Flournoy, JC, Hernandez, LM \& Dapretto, M 2019, 'Affective reactivity during adolescence: Associations with age, puberty and testosterone', Cortex, vol. 117, pp. 336350.

Weishaupt, D, Köchli, VD \& Marincek, B 2006, How Does MRI Work?, Springer Berlin Heidelberg, Berlin, Heidelberg.

Whittle, S, Simmons, JG, Byrne, ML, Strikwerda-Brown, C, Kerestes, R, Seal, ML, Olsson, CA, Dudgeon, P, Mundy, LK, Patton, GC \& Allen, NB 2015, 'Associations between early adrenarche, affective brain function and mental health in children', Social cognitive and affective neuroscience, vol. 10, no. 9, pp. 1282-1290.

Whittle, S, Yücel, M, Lorenzetti, V, Byrne, ML, Simmons, JG, Wood, SJ, Pantelis, C \& Allen, NB 2012, 'Pituitary volume mediates the relationship between pubertal timing and depressive symptoms 
during adolescence.', Psychoneuroendocrinology, vol. 37, no. 7, pp. 881-91.

Wierenga, LM, Bos, MGN, Schreuders, E, vd Kamp, F, Peper, JS, Tamnes, CK \& Crone, EA 2018, 'Unraveling age, puberty and testosterone effects on subcortical brain development across adolescence', Psychoneuroendocrinology, vol. 91, pp. 105-114.

Wierenga, LM, Langen, M, Oranje, B \& Durston, S 2014, 'Unique developmental trajectories of cortical thickness and surface area', Neurolmage, vol. 87, pp. 120-126.

Wong, AP-Y, Pipitone, J, Park, MTM, Dickie, EW, Leonard, G, Perron, M, Pike, BG, Richer, L, Veillette, S, Chakravarty, MM, Pausova, Z \& Paus, T 2014, 'Estimating volumes of the pituitary gland from T1-weighted magnetic-resonance images: Effects of age, puberty, testosterone, and estradiol', Neurolmage, vol. 94, pp. 216-221. 